\title{
Statues and Improvement of Electronic Medical Record System in Traditional Korean Medicine
}

\author{
Bo-Young Jung ${ }^{1+}$, Kyeong Han Kim ${ }^{2+}$, Song-Yi Kim ${ }^{3}$, Hyun-Kyung Sung ${ }^{4}$, Jeong-Su \\ Park ${ }^{5}$, Ho-Yeon $\mathrm{Go}^{6}$, Jang-Kyung Park ${ }^{7 *}$
}

\begin{abstract}
${ }^{1}$ Research Institute of Korean Medicine Policy, The Association of Korean Medicine, Korea
${ }^{2}$ Department of Preventive Medicine, College of Korean Medicine, Woosuk University, Korea

${ }^{3}$ Department of Anatomy and Acupoint, College of Korean Medicine, Gachon University, Korea

${ }^{4}$ Department of Pediatrics, College of Korean Medicine, Semyung University, Korea

${ }^{5}$ Department of Preventive Medicine, College of Korean Medicine, Semyung University, Korea

${ }^{6}$ Department of Internal Medicine, College of Korean Medicine, Semyung University, Korea

${ }^{7}$ Department of Obstetrics and Gynecology, College of Korean Medicine, Sangji University, Korea
\end{abstract}

\section{Key Words}

EMR, electronic medical record, health information, standardization

\begin{abstract}
Objectives: The study was to survey use of electronic medical records in subjects of Korean medicine doctors working for Korean medicine organizations and to contemplate ways to develop utilization of electronic medical records.
\end{abstract}

Methods: On August 2017, it conducted online self-reported survey on subjects of Korean medicine doctors at Korean hospitals and clinics who agreed to participate in the study. A total 40 doctors in hospital and 279 doctors in clinic were included. The surveyed contents include kinds of electronic chart, reason for not using electronic medical records and problems with creation of medical records.

Results: It finds that $100 \%$ of those working at Korean medicine hospitals and $86.4 \%$ of those at Korean medicine clinics have used electronic medical records. Sub-

Received: Aug 09, 2018 Reviewed: Aug 12, 2018 Accepted: Aug 22, 2018

$@$ This is an Open-Access article distributed under the terms of the Creative Common Attribution Non-Commercial License (http://creativecommons.org/licenses/by-nc/4.0/) which permits unrestricted noncommercial use, distribution, and reproduction in any medium, provided the original work is properly cited.

(2) This paper meets the requirements of KS X ISO 9706, ISO 9706-1994 and ANSI/NISO Z39.48-1992 (Permanence of Paper). jects answered the biggest reason for not using electronic medical records was inconvenience. The most serious problems with creation of electronic medical records at Korean medicine organizations found in the study include there was no method of creation of medical records and no standardized terminology for use in electronic medical records.

Conclusion: For utilization of electronic medical records at Korean medicine organizations, standardization of terminology, development of EMR in favour of its users and development of strategy that motivates use of EMR are required.

\section{Introduction}

As information technology in many are as rapidly advances, it has been growing demand for environment even in the medical field in which medical information was comprehensively managed and such information was shared among medical organizations to provide rapid and accurate diagnosis to patients regardless of time and place.

Regarding Paper Medical Record (PMR), it has problems in that it has a chance of record missing and errors depending on creators of medical records, easily making it incomplete information and becomes less

"Corresponding Author

Jang-Kyung Park. Department of Obstetrics and Gynecology, College of Korean Medicine, Sangji University, Usan-dong, Wonju-si, Gangwon-do, 26339, Korea.

Tel: +82-33-741-9275 Fax: +82-33-730-0679

E-mail: vivat314@naver.com 
available due to frequent occurrence of deterioration at its value or loss. To tackle these problems, Electronic Medical Record (EMR) was introduced. In 1999, the U.S. Institute of Medicine defined EMR as electronic type of record on patients included in hospital information system or prescription delivery system with which provides complete and accurate data on patients and assists doctors to make a clinical decision $[1,2]$.

In Korea, the medical law was revised in 2002 to contemplate legal grounds for introduction of EMR and enforced to create and store the electronic medical records with electronic signature. Such EMR introduction has been steadily on the increase. As of $2017,93.6 \%$ of conventional medicine hospital and $91.6 \%$ of conventional medicine clinic were found to use the EMR [3]. With increased use of the EMR for exchange of medical consultation information, the Korean government enacted 'standardization of medical consultation information for exchange' on standardized format of exchange, data format and method of transmission in 2017.

Korean medicine organizations were the active actor in introducing the EMR even though their participation was at lower level than conventional medicine institution. As of 2017, 85.9\% of TKM hospitals and $76.4 \%$ of TKM clinics used the EMR [1]. Because formats and terminologies used at Korean medicine organizations vary, there was difficulty in exchanging medical consultation records [4].

The study was to examine use of the EMR at Korean medicine organizations in terms of sharing medical consultation information among the organizations, and to contemplate ways to seek utilization.

\section{Methods}

\subsection{Participants}

It conducted online self-reported survey on subjects of Korean medicine doctors at Korean hospitals and clinics who agreed to participate in the study. The pool of those working at Korean medicine hospitals as participants was limited to clinical professors who work for 12 affiliated hospitals to Schools of Korean Medicine in the country and assign them in percentage quotas according to schools and medical fields. A survey on participants was conducted for two weeks starting from third to fourth week of August 2017. For those working at Korean medical clinics as participants, all the Korean medical doctors who were member of AKOM were sent an email to introduce the survey. The survey was conducted online on those who agreed on the participation. A survey on participants was conducted for 5 days from on August 21 to 25, 2017.

For those at the hospitals, a total of 40 subjects responded $(100 \%)$ No error or missing value in contents of response was found. For those at the clinics, a total of 436 subjects responded and among them, 124 subjects who were not working for the clinics and 33 subjects showed redundancy or error or missing value in their response were excluded to ultimately include 279 subjects (64.0\%), finally.

\subsection{Questionnaire}

Each draft of survey of questionnaires was developed by three Korean medicine experts based on the prior research results for those working at the hospitals and those at the clinics. A group of two clinical professors working at the hospitals and two Korean medicine doctors at the clinics reviewed each questionnaire two times for modification. For face validity, a pilot test was conducted on ten of them at the hospitals and ten of them at clinics to draw up with the final version of questionnaires. A complete survey of questionnaires was converted into electronic format online.

Study Variables.

The detailed variables were as follows.

(1) Demographic information: sex, age, clinic experience period, training.

(2) Status of usage of EMR

(3) Problems and Improvement of EMR

\subsection{Statistical Analysis}

Basic characteristics of the study sample were presented as frequencies and percentages. They were presented for each operational definition. Descriptive statistics were presented as type of care and hospital type. All statistical analyses were performed using SPSS version 21 for Windows (IBM Corp., Armonk, NY, USA).

\subsection{Ethics}

The survey was limited to those who read and show their agreement on research purpose, conducted anonymously and used only for academic purpose. The entire survey process was approved by the Institution Review Board of Semyung University Korean Medicine Hospital in Chungju (IRB number 1708-12).

\section{Results}

\subsection{Basic characteristics}

For gender, there was a higher number of males in both the hospitals and the clinics ( 28 males or $70.0 \%$ at the hospitals and 241 males or $86.4 \%$ at the clinics) and for age, subjects who in their forties were at the highest clinics (24 persons or $60 \%$ at the hospitals and 12 persons or $43.4 \%$ at the clinics).

For those at the hospitals, all the subjects were specialized medical doctors while 49 of them or $17.6 \%$ at the clinics participated. For clinical experience, there was the highest group of 29 or $72.5 \%$ at the hospitals and 116 or $41.6 \%$ at the clinics who have more than 15 years of clinical experience (Table 1). 
Table 1 Basic Characteristics of Respondents

\begin{tabular}{|c|c|c|c|c|c|}
\hline & \multirow{2}{*}{ Classification } & \multicolumn{2}{|c|}{ Hospital } & \multicolumn{2}{|c|}{ Clinic } \\
\hline & & $\mathrm{N}$ & $\%$ & $\mathrm{~N}$ & $\%$ \\
\hline \multirow{2}{*}{ Sex } & male & 28 & $70.0 \%$ & 241 & $86.4 \%$ \\
\hline & female & 12 & $30.0 \%$ & 38 & $13.6 \%$ \\
\hline \multirow{5}{*}{$\begin{array}{c}\text { Age } \\
\text { (years) }\end{array}$} & 20 's & 0 & $0.0 \%$ & 7 & $2.5 \%$ \\
\hline & 30 's & 11 & $27.5 \%$ & 93 & $33.3 \%$ \\
\hline & 40 's & 24 & $60.0 \%$ & 121 & $43.4 \%$ \\
\hline & 50 's & 5 & $12.5 \%$ & 53 & $19.0 \%$ \\
\hline & 60 's and over & 0 & $0.0 \%$ & 5 & $1.8 \%$ \\
\hline \multirow{12}{*}{ Training } & General Practitioner & 0 & $0.0 \%$ & 230 & $82.4 \%$ \\
\hline & Specialized Practitioner & 40 & $100.0 \%$ & 49 & $17.6 \%$ \\
\hline & Internal Medicine & 5 & $12.5 \%$ & 17 & $34.7 \%$ \\
\hline & $\begin{array}{l}\text { Acupuncture \& } \\
\text { Moxibustion }\end{array}$ & 5 & $12.5 \%$ & 9 & $18.4 \%$ \\
\hline & Gynaecology & 5 & $12.5 \%$ & 7 & $14.3 \%$ \\
\hline & $\begin{array}{l}\text { Sasang } \\
\text { Constitutional }\end{array}$ & 4 & $10.0 \%$ & 5 & $10.2 \%$ \\
\hline & Ophthalmology, & & & & \\
\hline & Otorhinolaryngology & 4 & $10.0 \%$ & 5 & $10.2 \%$ \\
\hline & \& Dermatology & & & & \\
\hline & $\begin{array}{l}\text { Rehabilitation } \\
\text { Medicine }\end{array}$ & 5 & $12.5 \%$ & 5 & $10.2 \%$ \\
\hline & Neuropsychiatry & 6 & $15.0 \%$ & 1 & $2.0 \%$ \\
\hline & Paediatrics & 6 & $15.0 \%$ & 0 & $0.0 \%$ \\
\hline \multirow{4}{*}{$\begin{array}{c}\text { Clinical } \\
\text { experience } \\
\text { period } \\
\text { (years) }\end{array}$} & under 5 & 0 & $0.0 \%$ & 36 & $12.9 \%$ \\
\hline & 5 and over to under 10 & 5 & $12.5 \%$ & 60 & $21.5 \%$ \\
\hline & 10 and over to under 15 & 6 & $15.0 \%$ & 67 & $24.0 \%$ \\
\hline & 15 and over & 29 & $72.5 \%$ & 116 & $41.6 \%$ \\
\hline
\end{tabular}


Table 2 Usage pattern of EMR

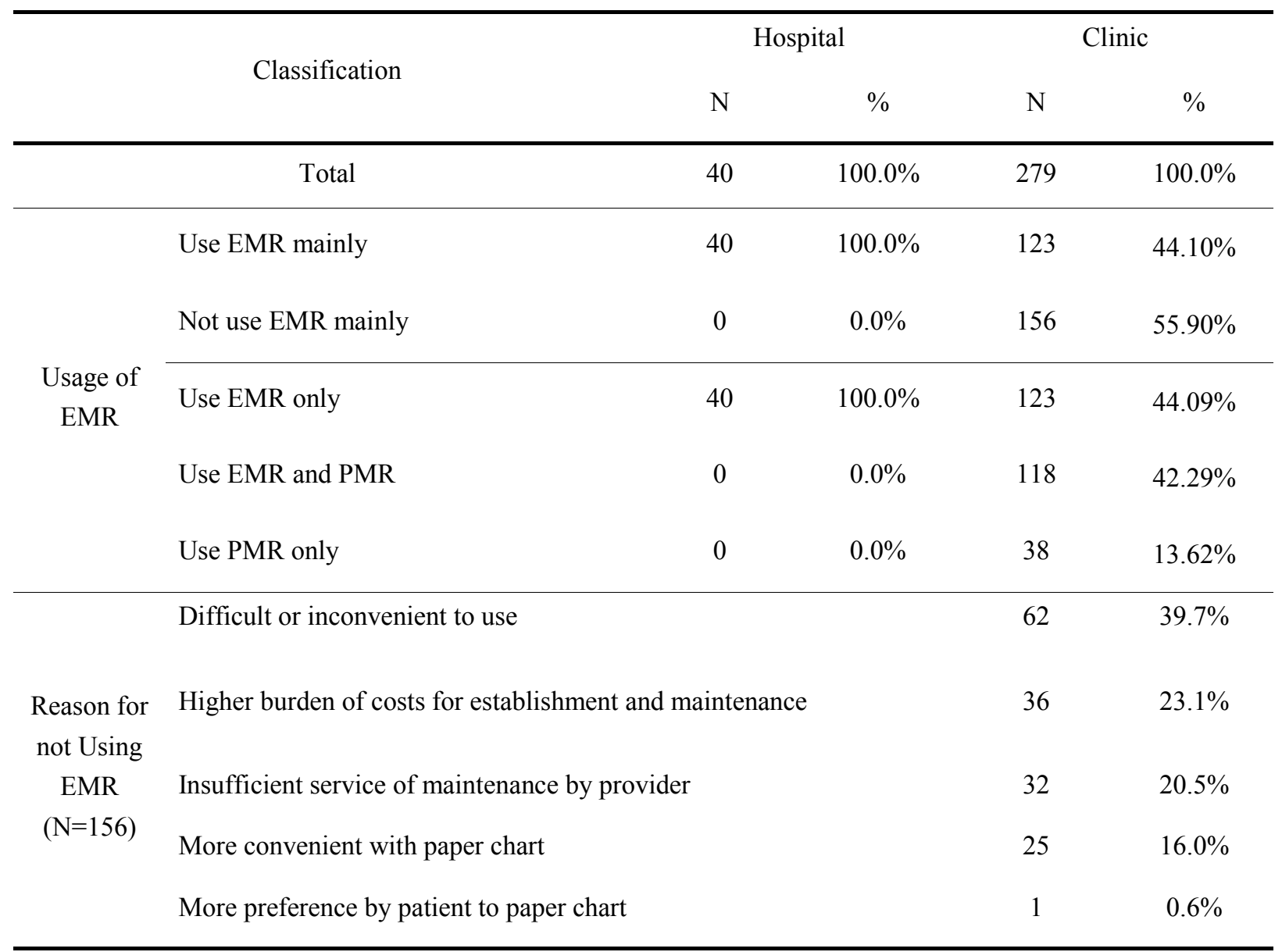

\subsection{Usage Pattern of EMR}

\subsubsection{Percentage of use of EMR and Reason for not using EMR}

A total of 40 subjects working for the Korean medicine hospitals all use the electronic medical records. Out of 279 subjects at the Korean medicine clinics, 123 persons or $44.1 \%$ use the EMR while 118 or $42.3 \%$ of them combine it with PMR. Each group of EMR users accounts for more than $40 \%$. Another group of PMR only users were 38 persons or $13.6 \%$, accounting for a slight number of $13.6 \%$.

For 156 subjects who do not use the EMR, 62 persons or $39.7 \%$ answered the reasons why they do not use it include that it was difficult and inconvenient to use, followed by 36 persons or $23.1 \%$ who responded cost burden for establishment and burdensome and insufficient maintenance service and with convenience of the paper chart (Table 2).

\subsubsection{Types of Charts in Use}

Korean medicine doctors working at the hospitals use the commercially used programs in which $27.6 \%$ use the OK Chart with the first largest share of percentage, followed by $26.1 \%$ using the Hanyi Maek with the second largest share of percentage and $18.3 \%$ Donguibogam of the third largest share of percentage (Table 3 ).

Four people working at the hospitals answered how the EMR was operated in Korean medicine hospitals. They reported one hospital used a self-developed program and the other three hospitals employed an externally developed program system. The employed externally developed program system was one production.

\subsection{The problem with creating medical records in Korean medicine}

In creation of medical records at Korean medicine organizations, those working for the hospitals were asked to raise the three highest ranked issues which include risk of missing information on non-insured medical consultation records, followed by no format of medical consultation records and the doctors using different terminology for the similar symptoms.

Those working for the clinics presumed the biggest prob- 
Table 3 Types of chart Using in Korean Medicine Clinic

\begin{tabular}{ccc}
\hline Name of Chart & $\mathrm{N}=241$ & $\%$ \\
OK Chart & 67 & $27.8 \%$ \\
Hanyi Meak & 63 & $26.1 \%$ \\
Donguibogam(Neobogam) & 44 & $18.3 \%$ \\
Hanyisarang & 22 & $9.1 \%$ \\
Han Chart & 19 & $7.9 \%$ \\
WinYeoyiju & 16 & $6.6 \%$ \\
Hanyi Chart & 5 & $2.1 \%$ \\
Dr. Hanyi & 1 & $0.4 \%$ \\
Doctors & 1 & $0.4 \%$ \\
Neo Chart & 1 & $0.4 \%$ \\
Hanbang Friend & 0 & $0.0 \%$ \\
Hanyicom & 0 & $0.0 \%$
\end{tabular}

lem with it would be much information missing from medical consultation records. Then, they pointed to possibility of omission of detailed information from the records, followed by unified format of records (Table 4).

Despite the concerns, an investigation on those subjects at the clinics for the current status of which medical consultation records were made found the similar level of creation in both insured medical consultation records and non-insured medical consultation records (Table 5).

\section{Discussion}

In the country, it has been widespread to require introduction of an electronic record at medical organizations as the information technology rapidly grows and the medical market changes, laying a foundation for medical informatization. Medical organizations provide a ubiquitous environment in which they can accumulate medical information and through the sharing of the information, make rapid and accurate diagnosis on patients regardless of time and place. There has recently been growing interest in EBM (evidence based medicine) in the country with analysis of the accumulated clinical information through EMR.

The study investigates use of the EMR in chart records by Korean Medicine doctors working for Korean Medicine organizations in order to contemplate ways to utilize the EMR.

We examine use and stages of the EMR by Korean Med- icine organizations, and evaluate results of the study against 5 stages of development proposed by the Medical Records Institute [11]. The results show that the clinical level of Korean Medicine organizations has variety of deviations including first to third stages while the hospital level of them stands at third stage. A study on fourth stage of EMR by Korean Medicine organizations remains its early stage. Korea Institute of Oriental Medicine developed OMS-Prime based on studies on diagnosis standardization [12], implemented a system that recommends demonstration through ontology inferences from disease symptoms and determine diagnosis according to the symptoms [13, 14]. The 5 staged electronic health record system includes all the health problems including not only medical issues of patents but also personal health. Kyung Hee University Hospital at Gangdong develops mobile organizer for atopy and makes first step toward development of Korean Medicine EMR.

There were three strategies that make a stride toward utilization of EMR at Korean Medicine organizations.

First, it requires infrastructure that was designed to standardize Korean Medicine terminology for utilization of the EMR at Korean Medicine. As the study found, the biggest problem with records of medical consultation at Korean Medical organization was that no standardization of formats and terminology in place. The standardization was made in both formats and contents. With no standardization yet in place, there was a limitation in representation of specialty in Korean Medicine that was so-called as empiricism [3]. The standardization in contents was 
Table 4 Problems in Creation of Medical Consultation Records at Korean Medicine institution

\begin{tabular}{|c|c|c|c|c|}
\hline \multirow{2}{*}{ Classification } & \multicolumn{2}{|c|}{ Hospital } & \multicolumn{2}{|c|}{ Clinic } \\
\hline & Score & Rank & Score & Rank \\
\hline Varying creation method of medical consultation records & 79 & 4 & 586 & 5 \\
\hline $\begin{array}{l}\text { Different terminology for the similar symptoms by } \\
\text { doctor }\end{array}$ & 93 & 3 & 630 & 4 \\
\hline Not sufficiently detailed records of medical consultation & 76 & 5 & 648 & 2 \\
\hline Missing information from medical consultation records & 99 & 1 & 721 & 1 \\
\hline No unified chart formats & 95 & 2 & 643 & 3 \\
\hline
\end{tabular}

* Total score was calculated with weight of $300 \%$ for the first, $200 \%$ for the second, and $100 \%$ for the third.

Table 5 Contents of Records in EMR program

\begin{tabular}{|c|c|c|c|}
\hline & Classification & $\mathrm{N}=241$ & $\%$ \\
\hline Treatment & National Insurance treatment & 171 & $70.95 \%$ \\
\hline Information & Non-insured treatment & 166 & $68.88 \%$ \\
\hline Payment & National Insurance payment & 154 & $63.90 \%$ \\
\hline Information & Non-insured payment & 163 & $67.63 \%$ \\
\hline
\end{tabular}

subject to diagnosis terminology, symptom terminology, treatment terminology and record method. The diagnosis terminology currently used by Korean Medicine doctors was KCD code that has mostly shown a similarity in that of physicians. When it comes to names of symptom and treatment as well as record method in the chart, however, no standardization was yet to be made. In view of prior studies published at the time of EMR utilization [7], the issue of standardization had continuously been reported even in physical medicine, and the subsequent efforts to develop such standardization in terminology for each field of the physical medicine have been aggressively made in pursuit of high quality of medical record [10]. In Korea, the Korea Standard Terminology of Medicine (KOSTOM) has been developed since 2009, established in 2014 on notifi- cation and then revised up to the present [9]. As Korean medicine terminology, part of acupuncture points which were established in $\ulcorner$ WHO Standard Acupuncture Point Locations in the Western Pacific Region」were notified as health terminology standardization of the country or KOSTOM in early 2018. Up until now, only Korean medicine terminology which notified as standardization was limited to acupuncture points. In the future, the standardization efforts for terminology should be on the way in order to utilize the EMR at Korean medicine organization.

Second, it was necessary to develop the EMR which adequately reflects needs on the Korean medicine clinics level for its utilization at all Korean medicine organizations. As of 2015, there were 260 Korean medicine hospitals (1.9\%) and 13,605 Korean medicine clinics (98.1\%) and the Kore- 
an medicine clinics represent the majority [5]. In the study, it was found that those at the clinics use much higher percentage of PMR or $55.9 \%$ compared to $0.0 \%$ at the hospitals. The reasons for not using the EMR include its difficulty and inconvenience in use and thus little affinity shared among the users (39.7\%), and cost burden for establishment and maintenance (23.1\%). In further study on the EMR utilization ratio to its establishment cost, the similar utilization ratio was shown between Hanyi Maek, the Korean medicine dedicated EMR solution of lower cost whose cost for establishment was free of charge and monthly maintenance cost 10,000 won and OK chart, more expensive option whose cost for establishment was 1,430,000 won and monthly maintenance cost 44,000 won. This would indicate that the establishment and maintenance costs were not the absolute factor in determining introduction of the EMR. A Korean medicine doctor who introduces the EMR shows a higher user affinity toward the more expensive OK chart. The reason he or she uses the OK chart was because it has customized functions such as patient reservation, consulting, statistics by condition and business information analysis, medicine inventory management and provision of medicine management information, and suggested prescription of the similar efficacy, and CDSS or clinical decision support system function. This would provide an indirect indication that those doctors who use the EMR have a higher demand for the unique function of medical records for diagnosis of patients' conditions, and the EMR needs for which he or she utilizes everything ranging from patient management, business analysis to clinical decision. The result shares a similarity with the findings in prior studies conducted on satisfaction of the EMR in conventional medicine clinic subjects that the use of the EMR would show a high satisfaction in that it makes the additional function to the required diagnosis function of patients easier [6]. Along with development of the EMR, there were needs for education targeting users and pathogenesis management which allow for continuous maintenance.

Third, the utilization of the EMR in Korean medicine requires accommodative strategies of motivation and provision at the clinical levels. It was presumed the reason for missing data records at Korean medicine organizations was their tendency to systematically avoid recording due to the high percentage of non-insured medical consultation [3]. In the study, however, no significant difference was found between the recording rates of insured medical consultation and non-insured medical consultation by $71 \%, 69 \%$ respectively. Therefore, there should be analysis to be followed that weighs benefits and losses in use of the EMR. To increase the EMR accommodating rates at Korean medicine organizations, the necessary education and promotion were required, for example, in ways that experience the EMR from clinical experiments or that educate of standardized terminology and creation of the electronic medical records targeting the doctors in their refresher training. In Korean medicine field, there was shortage of data source from which research materials can be used [8]. The EMR typed data accumulation can be utilized as secondary data because the data was able to be sorted as organized materials and thus increases its value as infor- mation [8]. The utilization of such information as research materials will largely reduce time and cost required for collection of research materials. It was an inevitable trend to utilize the EMR because it has its significance in making the first step toward reinforcing the basis for prescription and enhancing credibility of Korean medicines. The exchange of medical consultation data among Korean medicine organizations based on the EMR will make a contribution in that it shortens patients' waiting times, reduces unnecessary and redundant examination and promotes clinical approach based on empirical analysis of patient information and cooperative diagnosis across by physicians and Korean medicine doctors [7]. It was ultimately contributable in that the efforts would increase quality of medicine and provide the qualitative service of medicine by promoting operational efficiency and eventually making a delivery in efficient manner of the qualitative service to patients. .

\section{Conclusion}

The study on utilization of the EMR in Korean medicine field has limitations that it may generate a recall bias because the study analyzed results of survey that those responded made a self-filled response, and it may raise issues of representation because the subjects working for the hospitals were extracted in percentage quotas, instead of being randomly extracted. The study has a significance in that it suggests a direction for utilization of the EMR in Korean medicine field at a time when the social environment changes as aging population increases, and medical costs were on the rise, and there was a need for data analysis to make a policy proposal. It was necessary to have more closed study on standardization of the EMR for utilization in the future based on the drawn discussion from the study. The government, on its part, contemplates a policy to make an assistance with the EMR utilization while cultivating pool of its related trained and skilled manpower.

\section{Acknowledgment}

This study was supported by a grant of the Traditional Korean Medicine R\&D Project, Ministry of Health \& Welfare. Republic of Korea (HI 17C2187).

\section{Conflict of interest}

The authors declare that there are no conflicts of interest.

\section{References}

1. Rector A, Nowlan W, Kay S. Foundations for an electronic medical record. Methods Inf Med. 1991;30(3):179-86.

2. Powsner SM, Wyatt JC, Wright P. Opportunities for and challenges of computerisation. The Lancet. 1998;352(9140):1617-22.

3. HIRA. Publication of Results Regarding The Third Year 
Plan on Establishment and Utilization of Medical Consultation Data Exange Infrastructure; 2017.

4. Kyun Young-Kyu. DiagnosisStadardization of Chart Creation. Journal of Korean Medicine. 1994;15(2):30620.

5. AKOM. 2015 Yearbook of KIOM; 2015.

6. Koh S-J, Kim Y-S, Choi S-W, Yoon S-T. Anlaysis of Expectancy Effects of Establishment of Electronic Chart System at Clinics.

7. Park JS, Noh IC, Kim CS. Unied EMR Development for Cooperative diagnosis across the Medicines (Electronic Medical Records: EMR). Korea Business Science Society, Symposium Proceedings. 1999:139-40.

8. Korean Medicine Policy Research. Study on Measures of Utilization and Improvement of Public Data in Korean Medicine Fields; 2016.

9. Kim M. Comparative Analysis of SNOMED CT and Korea Standard Terminology of Medicine; 2016.

10. Jung BK, Kim J, Cho CH, Kim JY, Nam M-H, Shin $\mathrm{BK}$, et al. Report on the Project for Establishment of the Standardized Korean Laboratory Terminology Database, 2015. Journal of Korean medical science. 2017;32(4):695-9.

11. Gordon D, Geiger G, Lowe N, Jickling J, editors. What is an electronic patient record? Proceedings of the AMIA Symposium; 1998:American Medical Informatics Association.

12. Choi Seung-Hoon. Internet-based Hanyi Diagnosis Expert System (KHU-PIPE) developed; 2002.

13. Park Kyung-Mo, Park Jong-Hyun. Korean Medicine Methodology Describing Symptoms. Journal of Dongyi Physiology and Pathology. 2002;16(5):845-50.

14. Park JH, Shin SW, Jeong GS, Park KM, Kim SH. Hanyi Diagnosis Ontology Establishment and Evaluation. Journal of Dongyi Physiology and Pathology. 2006;20(1):202-8. 